\title{
Trust, Acceptance and Social Cues in Human-Robot Interaction (SCRITA)
}

\author{
Alessandra Rossi ${ }^{1}$ - Patrick Holthaus ${ }^{2}$ - Giulia Perugia ${ }^{3} \cdot$ Sílvia Moros ${ }^{2} \cdot$ Marcus Scheunemann $^{2}$
}

Published online: 1 December 2021

(C) The Author(s), under exclusive licence to Springer Nature B.V. 2021

The design of natural human-robot dynamics is a key aspect for ensuring a successful and efficient lasting human-robot interaction (HRI). In particular, it is expected that a robot deployed in human populated environments not only needs to be able to successfully complete a task, but also needs to show social intelligence to engage people in effective and natural interactions. In such scenarios, robots and people need to be able to cooperate to reach a joint goal, which can only be achieved if people accept and trust robots to complete their task and prevent any potential harm (emotional or physical) to people, the environment and themselves. A positive and balanced trust, indeed, is fundamental for building a highquality interaction. Moreover, robot should be capable of self-adapting to satisfy people's needs (i.e. personality, emotions, preferences, habits), and incorporating a reactive and predictive meta-cognition models to reason about the situational context (i.e. its own erroneous behaviours) and provide socially acceptable behaviours.

This special issue is composed by 16 manuscripts. The following collection of papers covers a wide range of topics of interests to identify some of the principal points to explore the role of trust in social robotics to effectively design and develop socially acceptable and trustable robots.

Alessandra Rossi

a.rossi@herts.ac.uk

Patrick Holthaus

p.holthaus@herts.ac.uk

Giulia Perugia

giulia.perugia@it.uu.se

Sílvia Moros

s.moros@herts.ac.uk

Marcus Scheunemann

m.scheunemann@herts.ac.uk

1 University of Naples Federico II, via Pansini, 5, 80131 Naples, Italy

2 University of Hertfordshire, Hatfield, England

3 Uppsala University, Uppsala, Sweden
The first paper presents the current state-of-art and challenges of healthcare robotics research by highlighting the communication behaviours to improve human-robot interactions. This review focusses on research in human-human social and clinical interactions, and compares them to the relevant perspectives and challenges in social robotics.

The second paper presents a review of the literature related to first encounters between robots and humans. It defines a taxonomy based on psychology and sociology models (Kendon's greeting model and Greenspan model of social competence) to categorise works that describe systems for robots to engage with people.

The third paper prepares a discussion about privacy issues regarding robots and older people in the home. This is accomplished with a new deck of cards that were created by the authors and then used by master students who interviewed 10 elderly people in their homes using these cards. Findings are split out by the cards as a method, how well specific components of the cards work, and the people's opinions about trust and robots as elicited by the cards.

The fourth paper proposes a model to predict people's trust in a robot in order to favour collaboration with users. Authors model the human agent's trust using a Beta distribution. The parameters for the distribution are estimated based on the robot's previous performance (failure or success) in personalised training trials. After the trials, humans only report their trust occasionally. The proposed approach complies with previously found properties on trust dynamics: (1) present trust is influenced by previous trust, (2) negative user experiences weight higher than positive, and (3) trust will stabilize over repeated interaction with the same agent.

The fifth paper presents a computational model that estimates user trust in an automated driving system, allowing for on-line trust measurements, and is tailored for use in autonomous vehicles. The model is verified for general feasibility and accurateness with a study where participants are asked to repeatedly self-report changes in trust towards the system during several trials. 
The sixth paper presents a study on proxemic functions for autonomous vehicles incorporating trust zones between a pedestrian and a driver, based on literature and validated in simulation and on public datasets. The research topic is relevant for the readers of this journal and the paper contributes with an extensive literature review and new insights.

The seventh paper presents an empirical study on how the movements of a non-humanoid robot affect the emotional responses and trust of human subjects. Results found that un-expected, non-social behaviours (such as moving in a negative manner or rejecting a participant's offer) can lead to an increase in anthropomorphic behaviour explanations.

In the eighth paper, authors report a solid meta-analysis of trust in child-robot interaction studies. The paper includes social and competence trust, interpersonal attitude, and likeability. The authors identify 20 suitable publications for the meta analysis, and it is a valuable contribution to the childrobot interaction research field, as it addresses theoretical and methodological challenges.

The ninth study presents a three parts study to investigate the role of touching gestures on people's trust in robots. Authors investigate self-reported ratings of a robot's trustworthiness and evaluation depending on contextual changes, i.e., gender of robot's voice, actor's gender, presentation of a picture showing a handshake, and functional framing, surrounding human-robot touch. The results reveal that observing touch can, on the one hand, result in higher ratings of trust-associated adjectives (studies $1+2$ ), but, on the other hand, decrease trust.

The tenth paper presents a two-studies work to investigate the effects of moral and immoral behaviours on people's social judgments of robots. Authors explores how evaluations of goodness or blame vary by moral foundations, and how these contribute to social evaluations of the agents. Results show that moral judgments do not differ across type of agent (human or robot), that all the moral foundations contribute to social evaluations and physical presence contributes to the assignment of blame/responsibility.

The eleventh paper presents the effects of agent's embodiment and eventual its incorrect behaviours over people's sense of loyalty and trust in a robot. In particular, when a robot willingly has hindering behaviours. Authors' findings highlight that the agent's embodiment does not affect the perception of quality of loyalty and the behvaiour of betrayal of an agent belonging to someone else. Furthermore, it shows that participants prefer embodied robot or disembodied voice to virtually agents under stressed situations.

The twelth paper provides a great contribution to socially assistive robots for assisting individuals with cognitive tasks, and how their non-verbal behaviours and communication styles can be altered to enhance individual's performance. More specifically, the authors studied the impact of using three different communications styles (friendly, neutral, and authoritarian) of the pepper robot on the cognitive performance of participants while the robot instructed them to complete a neuro-psychological evaluation.

The thirteenth paper relates a human's perceived level of control to their (negative) attitude towards a robot. Participants observed an interaction between a human and a robotic arm where the robot either behaved pro-actively or had a more passive role. The paper provides insights about the correlation between high levels of agency and negative attitudes.

The fourteenth paper consists of two studies. A preliminary $\mathrm{HH}$ study to infer which sets of verbal and non-verbal social cues used were associated to a more extraverted or introverted personality. In the second study, authors evaluated whether participants recognised same personality traits in a robot. Personality is an area of great importance in HRI, and research of this nature is fundamental for building a successful interaction.

The fifteenth paper describes an experimental study looking at the effect of consistent cooperative behavior on trust with an embodied virtual agent (EVA). The method presented provides a robust evaluation of the research question. Significant results provide a springboard for future research and EVA design. The study addresses an important topic with potentially wide-reaching applications to design of EVAs in many industries.

The last study establishes the relationship between the level of empathy in robotic speech and its perception in humans, and whether people prefer empathy in robotic voice synthesis. Authors evaluate a speech synthesizer capable of expressing primary and secondary emotions for the purpose of increasing acceptance among users through empathy. The study presented is a contribution that is useful to the HRI community.

\section{Additional Information}

This Special Issue is based on "Trust, Acceptance and Social Cues in Human Robot Interaction-SCRITA 2019" second edition Workshop that was held at the 28th IEEE International Conference on Robot and Human Interactive Communication-RO-MAN. The theme of this edition aims to explore different aspects of Human-Robot Interaction that can affect, enhance, undermine, or recovery humans' trust in robots, such as the use of social cues, behaviour transparency (goals and actions).

Publisher's Note Springer Nature remains neutral with regard to jurisdictional claims in published maps and institutional affiliations. 